\title{
Determinantes fisiológicos y ambientales de la regulación del control de la ingesta de alimentos
}

\author{
Physiological and environmental determinants of food intake regulation \\ Determinantes fisiológicos e ambientais da regulação do controle da ingesta de alimentos
}

\author{
Mónica Adriana Forero-Bogotá1*, Maritza Gómez Leguizamón²
}

Recibido: 21 de julio de 2020. Aceptado para publicación: 10 de octubre de 2020

Publicado en línea: 29 de octubre de 2020

https://doi.org/10.35454/rncm.v4n1.170

\section{Resumen}

La comprensión de factores que influyen sobre la regulación del apetito y la saciedad conduce al planteamiento de intervenciones en la prevención y manejo de enfermedades relacionadas con el exceso del consumo de alimentos. El objetivo de este artículo es realizar una revisión de los procesos fisiológicos y factores ambientales que intervienen como mecanismos de regulación de la ingesta alimentaria. Dicha regulación y el mantenimiento de la homeostasis del balance energético del organismo están mediados por múltiples factores. Se destacan los mecanismos fisiológicos, regulados por el hipotálamo mediante la señalización periférica y central, en la que intervienen señales de apetito o de saciedad. Además, con el creciente aumento del peso corporal, se destaca el cambio en el paradigma de las teorías homeostáticas que predominaban para explicar la regulación de la ingesta alimentaria, ya que se han quedado limitadas para definir el fenómeno del comportamiento alimentario. En este punto, los factores relacionados con el medio ambiente y con el comportamiento del individuo se vuelven relevantes para ilustrar el proceso. Por tanto, el estudio del tema debe ser abordado no solo desde la fisiología, sino también desde disciplinas de las ciencias sociales y comportamentales, porque es claro que la alimentación cumple tanto un rol fisiológico como un rol sociocultural. Asimismo, aspectos de

\section{Abstract}

The understanding of factors that influence appetite regulation and satiety leads to proposal of interventions aimed at preventing and managing diseases associated to overeating. The objective of this article is to review the physiological processes and environmental factors that control food intake. Regulation of food intake and maintenance of body energy homeostasis is mediated by multiple factors. Physiological mechanisms that mediate appetite or satiety signals and which are regulated by the hypothalamus through peripheral and central signaling, are highlighted. Moreover, with an increase in body weight, the change in the paradigm of homeostatic theories aimed at explaining the food intake regulation of is highlighted, as they have been limited to define the phenomenon of food behavior. This is where environmental and behavioral factors become relevant to explain the process. Therefore, the study of food consumption must be addressed not only from a physiological perspective, but also from a social and behavioral perspective, as it is evident that food not only fulfills a physiological role, but also a social and a cultural one. Likewise, aspects of modernity such as sedentary behavior, industrial food processing and the wide offer of them, play a decisive role in the regulation of appetite and satiety.

\section{Resumo}

A compreensão dos fatores que influenciam na regulação do apetite e a saciedade conduzem ao planejamento de intervenções na prevenção e manejo de doenças relacionadas com o excesso do consumo de alimentos. $\mathrm{O}$ objetivo deste artigo foi realizar uma revisão dos processos fisiológicos e dos fatores ambientais que intervêm como mecanismos de regulação da ingestão alimentar.

A regulação da ingestão alimentar e a manutenção da homeostase do balanço energético do organismo são mediadas por múltiplos fatores. Destacam-se os mecanismos fisiológicos regulados pelo hipotálamo por meio de sinalização periférica e central, mediando sinais de apetite ou saciedade. Porém, com o aumento crescente do peso corporal, destaca-se a mudança de paradigma das teorias homeostáticas que predominaram para explicar a regulação da ingestão alimentar, uma vez que se limitaram a definir o fenômeno do comportamento alimentar.

É aqui que fatores relacionados ao meio ambiente e ao comportamento do indivíduo são relevantes para ilustrar o processo. Portanto, o tema de estudo deve ser abordado no só desde o campo da fisiologia, senão desde as disciplinas das ciências sociais e comportamentais, pois é evidente que a alimentação não só cumpre um papel fisiológico, senão também um papel social e cultural. Além disso, aspectos da modernidade como es- 
la modernidad como el comportamiento sedentario, el procesamiento industrial de alimentos y la amplia oferta de los mismos juegan un rol decisivo en la regulación del apetito y la saciedad.

Palabras clave: apetito, saciedad, regulación del apetito, consumo de alimentos, hambre.
Keywords: Appetite; Satiation; Appetite regulation; Food consumption; Hunger.
Grupo de Nutrición Cardiovascular de la Sociedad Colombiana de Cardiología y Cirugía Cardiovascular. Bogotá, Colombia.

*Correspondencia: Mónica Adriana Forero Bogotá monicaabogota@gmail.com

\section{INTRODUCCIÓN}

Las necesidades nutricionales de un individuo están determinadas por factores como edad, sexo, peso, talla, nivel de actividad física y composición corporal. Estas deben ser cubiertas mediante la ingesta de variedad de alimentos, de manera que se garantice un balance nutricional y energético que priorice la promoción de la salud y la prevención de la enfermedad.

Desde 1975 hasta la fecha las cifras del exceso de peso se han triplicado en el mundo. De acuerdo con cifras de la Organización Mundial de la Salud (OMS), en 2016 el $39 \%$ de la población mundial mayor de 18 años presentaba sobrepeso y el $13 \%$, obesidad; y en ambos casos era mayor en mujeres que en hombres ${ }^{(1)}$. Colombia no es ajena a esa realidad: de acuerdo con la Encuesta Nacional de Salud y Nutrición (ENSIN) de 2015 , el $37,8 \%$ de la población presentaba sobrepeso y el $18,7 \%$, obesidad ${ }^{(2)}$. Si se considera que el exceso de peso corresponde a una entidad multifactorial en la cual intervienen factores genéticos, ambientales, endocrinos, metabólicos, conductuales, emocionales, psicológicos, comportamentales, entre otros ${ }^{(3)}$, un área de investigación importante corresponde al estudio de los mecanismos que regulan el hambre y la saciedad; este se convierte en un componente relevante para la comprensión del aumento excesivo de peso en la población mundial.

Actualmente, se sabe que la regulación en la ingesta de alimentos no solo está mediada por factores endógenos centrales y periféricos ${ }^{(4,5)}$, sino también por factores como la composición de macronutrientes de la $\operatorname{dieta}^{(6)}$, la actividad física ${ }^{(7)}$ y por factores ambientales tilo de vida sedentário, o processamento industrial de alimentos e ampla oferta dos produtos, desempenham um papel decisivo na regulação do apetite e a saciedade.

Palavras-chave: apetite, saciedade, regulação do apetite, consumo de alimentos, fome.
2 Grupo de Investigación Rendimiento Físico Militar (RENFIMIL), Escuela Militar de Cadetes “General José María Córdova”. Bogotá, Colombia. como la exposición a alimentos con carácter hedónico y altamente agradables ${ }^{(8)}$, la textura de los $\operatorname{mismos}^{(9,10)}$, masticación o procesamiento oral de las comidas ${ }^{(11)}$, tamaño de los platos y de la porción del alimento, entre otros $^{(12,13)}$

Este artículo tiene como objetivo hacer una revisión sobre los componentes fisiológicos, ambientales, alimentarios, comportamentales, psicológicos y hedónicos que intervienen como mecanismos de regulación de la ingesta de alimentos. Para ello se realizó una búsqueda en bases de datos como Medline, PubMed y Scopus, en la que se usó terminología $\mathrm{MeSH}$ relacionada con el tema y se usaron operadores booleanos; se consideraron artículos tanto en inglés como en español publicados entre los años 2010 y 2020.

\section{MECANISMOS DE REGULACIÓN FISIOLÓGICA DE LA INGESTA DE ALIMENTOS}

La alimentación es un proceso transversal a la vida del ser humano, que está mediado por componentes fisiológicos y ambientales. La sensación de hambre se relaciona con la necesidad urgente de ingerir alimentos, influida además por señales fisiológicas como hipoglucemia, contracciones gástricas y aumento de ruidos intestinales ${ }^{(14-17)}$. El apetito, por su parte, corresponde al deseo psicológico por comer alimentos concretos, más asociado con experiencias sensoriales y emotivas, y sin asociación con las anteriores señales fisiológicas de hambre ${ }^{(18)}$. Si el consumo de alimentos tiene efecto, sobreviene la señal de satisfacción o plenitud, conocida como saciación, experimentada durante o inmediatamente después de la ingesta de los alimentos, que invita a dejar de comer; y la 
saciedad, que hace referencia a un período más prolongado sin hambre o sin necesidad de requerir alimentos hasta la siguiente señal de hambre ${ }^{(17,18)}$.

\section{¿QUÉ CONTROLA LA INGESTA ALIMENTARIA?}

La regulación de la ingesta alimentaria es mediada por un complejo mecanismo en el que participa el hipotálamo, el cual es el encargado de regular las señales de hambre y saciedad. Estas señales permiten alcanzar la homeostasis de la energía ${ }^{(19)}$ a través de sus estructuras, como lo son el núcleo lateral (responsable de la sensación de hambre) y el núcleo ventromedial (que se encarga del manejo y control de la sensación de saciedad durante el proceso de la ingesta de alimentos). Los núcleos paraventriculares, dorsomediales y arqueados del hipotálamo también contribuyen a la regulación de la ingesta de alimentos e influyen en la producción de hormonas tiroideas, suprarrenales, insulina y glucagón, como también grelina y leptina. De esta manera, tiene lugar la regulación de la ingesta y el balance energético a corto, mediano y largo plazo $^{(14)}$ (Figura 1).

\section{REGULADORES GASTROINTESTINALES, NEUROENDOCRINOS Y PERIFÉRICOS}

El hipotálamo recibe señales del sistema gastrointestinal a través del nervio vago, las cuales informan sobre aspectos como el llenado gástrico. Así mismo, se envían señales químicas sobre la presencia de nutrientes a nivel sanguíneo (glucosa, aminoácidos, ácidos grasos) que pueden determinar la señal de saciedad.

En cuanto a los estímulos de hambre u orexígenos a nivel periférico, en primera instancia se reconoce a la grelina, un neuropéptido producido en el estómago, en las células del fondo gástrico; y la motilina, una hormona sintetizada por el duodeno ${ }^{(5,19)}$. La grelina tiene la capacidad de atravesar la barrera hematoencefálica y actúa sobre el núcleo arcuato, donde activa receptores

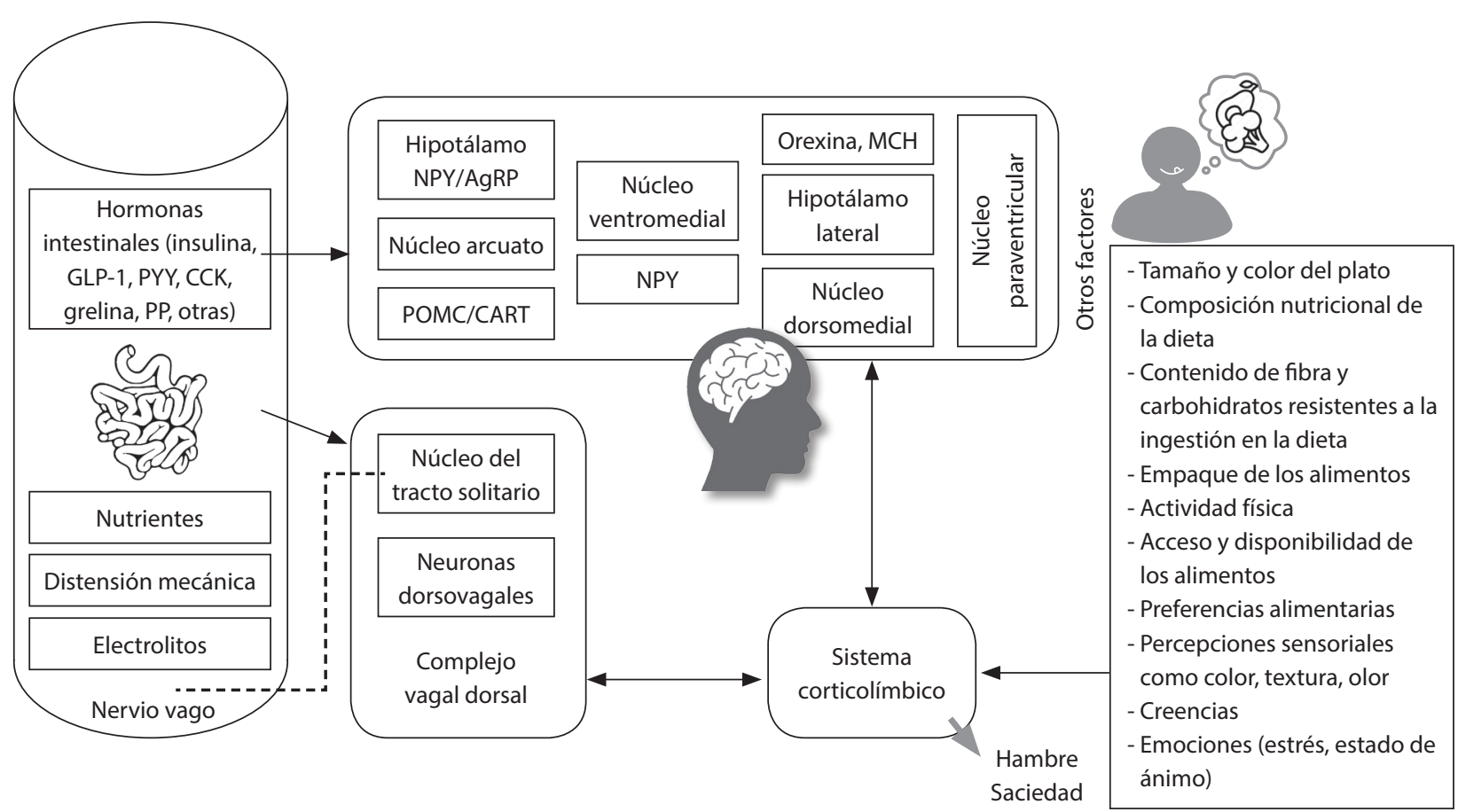

Figura 1. Mecanismos homeostáticos y otros factores que regulan la ingesta alimentaria. Fisiológicamente, el hambre está regulada por mediadores neuroendocrinos, gastrointestinales y periféricos que actúan a nivel hipotalámico y regulan la sensación de hambre o saciedad. De este modo, los mediadores producidos a nivel intestinal como la insulina, el péptido similar al glucagón (GLP-1), el péptido Y (PYY), la colecistoquinina (CCK), la grelina, el polipéptido pancreático (PP), entre otros, determinados en parte por la calidad y cantidad de comida ingerida, envían información al hipotálamo a través del nervio vago estimulando los centros del hambre o la saciedad y la consecuente producción de neuropéptidos como el neuropéptido Y (NPY), la orexina, el péptido relacionado con la proteína Agouti (AgRP), entre otros. Por su parte, se reconocen otros factores relacionados con el medio ambiente como las emociones, características hedónicas que influyen sobre la sensación de hambre o saciedad. Modificado de ${ }^{(8)}$. 
relacionados con la conducta alimentaria como el NPY y el péptido relacionado con la proteína Agouti (AgRP) (14), los cuales aumentan la ingesta alimentaria y reducen el metabolismo energético, así como la hormona concentradora de melanina $(\mathrm{MCH})$, producida en el hipotálamo lateral. La grelina, también conocida como la hormona del hambre, estimula la búsqueda e ingesta de alimento tanto en individuos sanos como en obesos. Aunque este es el papel más conocido de la grelina, también tiene funciones sobre el ciclo del sueño-vigilia, regula el metabolismo de la glucosa ${ }^{(20)}$, interviene en la motilidad intestinal acelerando el vaciamiento gástrico, induce adiposidad y está relacionada con el metabolismo energético ${ }^{(5)}$. Por estas razones, es de gran interés, junto con la leptina, en el estudio de la regulación del peso corporal y la obesidad.

El NPY se sintetiza directamente en el hipotálamo a nivel del núcleo arcuato; su efecto es directamente a nivel central, donde estimula y potencia la ingesta de alimentos y, en muchos casos, la ganancia de peso. Tanto la grelina, como el NPY y la AgRP son inhibidos por señales de saciedad derivadas de la leptina, la insulina y la glucosa ${ }^{(19)}$.

Dentro de los mecanismos que controlan la ingesta alimentaria a nivel periférico, se han descrito la producción de la CCK y el PYY, los cuales son producidos en el estómago luego de la entrada de los alimentos al mismo; envían una señal periférica a través del nervio vago que termina en el núcleo del tracto solitario y el núcleo parabraquial, lo que disminuye la ingesta de alimentos $^{(21)}$. El GLP1 (liberado por las células del intestino en respuesta a la ingesta de alimentos), junto con la oxintomodulina (también derivada del proglucagón, al igual que el GLP1) inducen saciedad. La oxintomodulina también ha mostrado efectos sobre el aumento del gasto energético y la reducción del peso corporal ${ }^{(19,22)}$.

La leptina cumple un rol opuesto a la grelina. Esta hormona, liberada por el tejido adiposo, actúa a nivel hipotalámico, reduciendo la ingesta de alimentos y aumentando el gasto energético ${ }^{(19)}$. En situaciones normales, la leptina se libera al torrente sanguíneo y señala al cerebro el estado de las reservas energéticas del organismo; razón por la cual la leptina es de gran interés, ya que tiene una enorme influencia en la regulación del peso corporal. Es importante aclarar que la leptina no solo cumple un rol en la regulación energética, sino también que su producción varía en función de la ingesta de alimentos, el sexo, la edad, el ejercicio y la glucosa circulante ${ }^{(20)}$. Cabe mencionar que existen mecanismos fisiopatológicos en los cuales se presenta una reducción de la sensibilidad de los tejidos a la leptina, denominada resistencia a la leptina; es el caso de individuos obesos en los cuales, a pesar del aumento en las concentraciones de leptina, el efecto anorexígeno de la misma no es el esperado ${ }^{(23,24)}$.

En condiciones normales, la insulina, liberada por el páncreas, actúa a nivel del núcleo arcuato y cuerpo paraventricular en el hipotálamo: disminuye la ingesta y aumenta el gasto de energía. De este modo, la insulina comparte algunas similitudes con la leptina ${ }^{(14)}$.

Como se relaciona anteriormente, el intestino y el tejido adiposo juegan un papel crucial en la señalización del control del hambre y la saciedad, ya sea propiciando señales de hambre que inviten al individuo a ingerir alimentos o, por el contrario, reduciendo el consumo de los mismos ${ }^{(20)}$.

\section{OTROS FACTORES QUE DETERMINAN LA INGESTA DE ALIMENTOS}

Reducir el control del hambre y la saciedad a factores fisiológicos no permitiría tener un panorama amplio sobre la conducta alimentaria de los individuos, y sería aún más difícil comprender el fenómeno de la obesidad.

De acuerdo con la literatura, como se expone más adelante, existen múltiples factores ambientales y comportamentales que determinan la ingesta de alimentos.

\section{Sueño}

Se han presentado varias explicaciones para describir el vínculo entre el sueño y la mayor ingesta de alimentos, y por consiguiente, la obesidad ${ }^{(25)}$ :

- El tiempo adicional despierto, que aumenta las oportunidades para comer;

- El aumento de la grelina, con su posterior estímulo del centro del apetito y la reducción de las hormonas que promueven la saciedad;

- La termorregulación alterada; y

- El aumento de la fatiga, lo que se traduce en menor nivel de actividad física ${ }^{(6)}$.

También se ha sugerido que las alteraciones en el horario del sueño y las comidas, junto con la duración del sueño, podrían influir en los niveles hormonales relacionados con la saciedad y la ingesta de alimentos ${ }^{(26)}$, así como conllevar a alteraciones en el control de la glucemia y resistencia a la insulina ${ }^{(27)}$, lo que finalmente conduce a un aumento del hambre y posterior ingesta de alimentos. 


\section{Tamaño y color del plato}

De acuerdo con lo descrito por A. McClain y colaboradores, servir en platos más chicos puede ayudar visualmente al cerebro. Según datos y observaciones, en los últimos 30 años el tamaño de los platos aumentó en un $36 \%$; probablemente el aumento del tamaño promedio de los platos usados para servir los alimentos se traduce en porciones más grandes ${ }^{(12)}$ y mayor ingesta calórica, lo que se asocia con el sobrepeso y la obesidad ${ }^{(13,28)}$. Asimismo, en una investigación realizada en adultos se identificó que los individuos se sirvieron más comida cuando esta y los platos tenían un color similar (bajo contraste); y, por el contrario, se sirvieron menos comida cuando esta y los platos eran de diferentes colores (alto contraste) ${ }^{(13)}$.

\section{Composición nutricional de la dieta}

El rol atribuido a los nutrientes en las señales de hambre y apetito, y la ingesta de energía han cambiado con el tiempo. Los productos digestivos o los metabolitos circulantes de los nutrientes se han visto como:

- Señales para iniciar eventos alimenticios, determinando así la frecuencia de la alimentación;

- Señales para terminar eventos ingestivos, controlando así el tamaño de la porción; y

- Señales que activan los sistemas de recompensa cerebral que pueden desregular una alimentación saludable ${ }^{(6)}$.

Durante las últimas décadas, ninguna dieta para perder peso ha sido ampliamente exitosa, probablemente debido al fracaso para abordar adecuadamente los efectos de la frecuencia de las comidas y el tamaño de las porciones al mismo tiempo, así como el hecho de que el comportamiento ingestivo está guiado por muchos factores cognitivos y ambientales ${ }^{(17)}$.

Respecto a la distribución de macronutrientes en la dieta, en un estudio preliminar realizado por L. Tischmann y colaboradores ${ }^{(29)}$ identificó que una dieta alta en proteínas y moderada en carbohidratos ( $25 \%$ y $45 \%$, respectivamente), comparada con una dieta más alta en carbohidratos y normal en proteína (55\% y 15 $\%$, respectivamente), redujo la percepción de hambre en el grupo de estudio, ya que una dieta alta en proteína y moderada en carbohidratos promueve mayores concentraciones posprandiales de PYY y 2 -araquidonilglicerol $(2-A G)^{(29)}$. Sin embargo, los aportes altos de proteína no han demostrado ser sostenibles a largo plazo en una gran proporción de la población ${ }^{(6)}$.

\section{Señales sensoriales como color, textura, olor y empaque de los alimentos}

La experiencia sensorial, vinculada a aspectos como el color, la textura y el olor ${ }^{(30)}$, así como asociaciones emotivas y de la memoria con los alimentos, comidas o preparaciones también determinan la selección y posterior ingesta de alimentos ${ }^{(31)}$. De acuerdo con lo descrito por K. McCrickerd y colaboradores ${ }^{(30)}$, las características sensoriales de los alimentos pueden determinar las preferencias, el tamaño de la porción y la sensación de saciedad, e incluso facilitar un aprendizaje dietético ${ }^{(32)}$. Por ejemplo, la visualización de un alimento en una proximidad cercana al individuo es la primera señal o invitación para su consumo, y la apariencia de ese alimento o comida puede maximizar su consumo; de este modo, algunos autores han descrito el sesgo de la unidad ${ }^{(10)}$, es decir, una misma porción de un alimento ofrecida en porciones más chicas o piezas fraccionadas parece ofrecer mayor saciedad, sin cambiar el placer por el mismo.

Asimismo, se ha identificado que aspectos como la temperatura ambiental, la luz, el sonido o la realización de actividades alternativas como ver televisión o trabajar en el computador pueden influir sobre la ingesta de alimentos y la capacidad de respuesta o percepción de saciedad $^{(8,17,33-35)}$.

\section{Contenido de fibra y carbohidratos resistentes a la digestión en la dieta}

Los granos integrales, las frutas y las verduras han demostrado su alto potencial para reducir el riesgo de obesidad, enfermedades cardiovasculares y diabetes tipo 2. Un posible mecanismo detrás de los beneficios del grano integral, específicamente, es la fermentación intestinal de la fibra dietética ${ }^{(36)}$. Revisiones sistemáticas realizadas han demostrado que la fibra tiene un efecto benéfico sobre la regulación del apetito y el control de la ingesta de energía ${ }^{(37)}$. Una de estas características es la regulación hormonal a través del consumo de fibra dietética, al favorecer la producción de ácidos grasos de cadena media, los cuales estimulan la producción de hormonas supresoras del apetito, como el GLP 1 y el PYY ${ }^{(38)}$. De este modo, en la medida en que varias hormonas del apetito $y$ la saciedad se activen ante la presencia de fibra dietética, se proporcionará un efecto de saciedad que causará una disminución en el consumo de alimentos ${ }^{(39,40)}$; esto se puede considerar una herramienta útil para la regulación de la ingesta energética. 


\section{Rol de la actividad física}

Cuando las personas cambian de un estado activo a un estado sedentario y no hay la regulación negativa de la ingesta de alimentos, consecuente a la reducción del gasto de energía, el resultado será un balance energético positivo y el potencial aumento de peso ${ }^{(41)}$.

Se ha demostrado una respuesta hormonal aguda frente al ejercicio en la cual, después de la realización de ejercicio de resistencia superior al $60 \%$ del consumo de oxígeno $\left(\mathrm{VO}_{2}\right)$ pico, se observa una percepción del apetito reducida durante los 30 a 60 minutos posteriores a la actividad; esto se ha denominado anorexia inducida por el ejercicio, un efecto inducido por una supresión en los niveles de grelina y elevaciones en las concentraciones de GLP1 y PYY. Respecto a los efectos de la actividad física crónica, aunque la evidencia es contradictoria, la realización de ejercicio de manera crónica produce un equilibrio entre un aumento en el impulso hacia la ingesta de alimentos, derivado de un mayor gasto energético, que a su vez es balanceado con una mayor satisfacción o plenitud ante una comida y mayor saciedad después de la misma ${ }^{(42)}$.

\section{Rol hedónico de los alimentos o comidas}

De acuerdo con HM. Espel-Huynh y colaboradores, el término apetito hedónico se refiere a la preocupación y el deseo de consumir alimentos con fines de placer y en ausencia de hambre física ${ }^{(43)}$. Entonces, la conducta alimentaria no está regulada solamente por mecanismos homeostáticos, sino que también intervienen otros factores de tipo emocional, sensorial, mecánico y cognitivo que han sido identificados y, en conjunto, se han denominado sistema hedónico ${ }^{(8)}$. Este sistema se activa mediante mecanismos de recompensa y experiencias frente a los alimentos, que independientemente de su valor nutricional ofrecen al consumidor placer y gratificación; estos alimentos suelen ser densos en calorías y ricos en azúcar, sal y grasas. El impulso por consumir estos alimentos puede incluso anular las señales fisiológicas, favoreciendo un desbalance en el equilibrio energético. Por tanto, la recompensa alimentaria sería la mayor impulsora para la ingesta de alimentos, más allá de la sensación de apetito o saciedad. La palatabilidad de los alimentos hedónicos está influenciada por características organolépticas como olor, textura, color, $\mathrm{o}$, incluso, sonido emitido ${ }^{(8)}$.

En la Figura 1 se resumen los mecanismos homeostáticos y demás factores ambientales que intervienen en la regulación de la ingesta alimentaria.

\section{DISCUSIÓN}

La ingesta de alimentos es modulada por varios mecanismos homeostáticos y no homeostáticos que controlan el hambre. Extensos esfuerzos de investigación en los últimos 30 años han revelado gran parte de los detalles moleculares y neuroanatómicos del control del equilibrio de energía, involucrando el tracto gastrointestinal, péptidos intestinales, nervios periféricos y sistemas neuroendocrinos y de recompensa en el cerebro, y cómo interactúa la comida con estos sistemas y procesos ${ }^{(15)}$. Así como la alteración del sueño se ha asociado con la resistencia a la leptina y el consiguiente aumento de los niveles de esta proteína, lo que resulta en un aumento del hambre ${ }^{(44)}$, existen estudios publicados que proporcionan evidencia de que las sensaciones de hambre y saciedad dependen de la dosis de sueño; asimismo, se asocian fuertemente con el consumo de almidones resistentes ${ }^{(45)}$ y alimentos altos en fibra, como granos enteros ${ }^{(46)}$, que aumentan la percepción de saciedad y reduce los índices de hambre.

De igual manera, se ha evidenciado que en la percepción de hambre en sujetos con niveles altos de actividad física hay un control más sensible ${ }^{(47)}$. La actividad física afecta los mecanismos homeostáticos del hambre y la saciedad, a través de una acción de proceso dual con un mayor gasto energético y una mayor respuesta de saciedad a los alimentos, probablemente a través de una señalización posprandial más sensible. En una consideración amplia del control del hambre y el apetito, se pueden distinguir tres cuestiones: los orígenes del impulso de comer (apetito), la elección de alimentos y las decisiones sobre qué comer, y el control sobre la inhibición de comer y la cantidad de comida consumida (cuánto se come). A su vez, la obesidad puede surgir de un fuerte impulso para comer, elecciones de alimentos inapropiados (por ejemplo, alimentos con fuerte atractivo sensorial y alta densidad de energía) o una inhibición débil de la alimentación. Las personas varían notablemente en la fuerza y dirección de estos procesos ${ }^{(48)}$.

Por su parte, una mayor disponibilidad y exposición a los alimentos altamente agradables, y la oportunidad de elegir alimentos casi ilimitados hace que el ser humano sea vulnerable al consumo excesivo y la obesidad. Por tanto, es importante que los investigadores y los médicos tengan acceso a procedimientos para la medición confiable y válida del impulso de comer (principalmente el apetito y las sensaciones asociadas), procesos inhibitorios sobre la alimentación (saciación y saciedad) y mecanismos que conducen a diferentes 
opciones de alimentos (por ejemplo, los atributos hedónicos de gustar y desear).

\section{CONCLUSIONES}

Comer forma parte de una cultura donde el acceso a los alimentos es cómodo, principalmente a alimentos altamente agradables. Reconocer que la regulación del apetito y la saciedad no están mediadas solo por mecanismos fisiológicos u homeostáticos permite al profesional en nutrición y dietética considerar otros aspectos relacionados más con el contexto del individuo y el ambiente en el cual este se desarrolla, con el objetivo de integrar herramientas que le permitan identificar en qué sistema está la desregulación y plantear soluciones al mismo.

La presentación implícita y explícita de los alimentos puede determinar el consumo de los mismos, más allá de una necesidad fisiológica de su consumo. Esto incluye evaluación de los factores del estilo de vida, un historial médico pasado y presente detallado, el uso de medicamentos o suplementos, un resumen global del entorno sociocultural y un examen físico completo.

\section{Financiación}

Para la realización de este artículo de revisión no se recibió financiación de ninguna entidad ni pública ni privada.

\section{Conflicto de intereses}

Los autores declaran no poseer ningún tipo de conflicto de interés.

\section{Declaración de autoría}

Las autoras participaron en la concepción, búsqueda y desarrollo de la revisión; así como también en la redacción del artículo. Todos los autores revisaron el artículo y validaron su versión final.

\section{Referencias bibliográficas}

1. Obesidad y sobrepeso [Internet]. Organización Mundial de la Salud (OMS); 2018. [Consultado el 6 de mayo de 2019]. Disponible en: https://www.who.int/es/news-room/factsheets/detail/obesity-and-overweight.

2. Encuesta Nacional de Alimentación y Nutrición. ENSIN 2015 nota de política. [Internet]. Ministerio de Protección Social, Instituto Colombiano de Bienestar familiar; 2015 [consultado el 2 de junio de 2020]. Disponible en: https:// www.icbf.gov.co/bienestar/nutricion/encuesta-nacionalsituacion-nutricional.
3. Suárez-Carmona W, Sánchez-Oliver AJ, González-Jurado JA. Pathophysiology of obesity: Current view. Rev Chil Nutr. 2017;44(3):226-33.

4. Wen S, Wang C, Gong M, Zhou L. An overview of energy and metabolic regulation. Sci China Life Sci. 2019;62(6):771-90. doi: 10.1007/s11427-018-9371-4.

5. Abdalla MMI. Central and peripheral control of food intake. Endocr Regul. 2017;51(1):52-70. doi: https://doi. org/10.1515/enr-2017-0006.

6. Carreiro AL, Dhillon J, Gordon S, Jacobs AG, Higgins KA, McArthur BM, et al. The Macronutrients, Appetite, and Energy Intake. Annu Rev Nutr. 2016;36:73-103. doi: 10.1146/annurev-nutr-121415-112624.

7. Hopkins M, Blundell JE. Energy balance, body composition, sedentariness and appetite regulation: Pathways to obesity. Clin Sci. 2016;130(18):1615-28. doi: 10.1042/CS20160006.

8. Hernández Ruiz de Eguilaz M, Martínez de Morentin AB, Almiron-Roig E, Pérez-Diez S, San Cristóbal Blanco R, NavasCarretero S, et al. Influencia multisensorial sobre la conducta alimentaria: ingesta hedónica. Endocrinol Diabetes Nutr. 2018;65(2):114-25. doi: 10.1016/j.endinu.2017.09.008.

9. Mosca AC, Torres AP, Slob E, de Graaf K, McEwan JA, Stieger M. Small food texture modifications can be used to change oral processing behaviour and to control ad libitum food intake. Appetite. 2019;142:104375. doi: https://doi.org/10.1016/j. appet.2019.104375.

10. Marchiori D, Waroquier L, Klein O. Smaller Food Item Sizes of Snack Foods Influence Reduced Portions and Caloric Intake in Young Adults. J Am Diet Assoc. 2011;111(5):72731. doi: 10.1016/j.jada.2011.02.008.

11. Ferriday D, Bosworth ML, Godinot N, Martin N, Forde CG, Van Den Heuvel E, et al. Variation in the oral processing of everyday meals is associated with fullness and meal size; A potential nudge to reduce energy intake? Nutrients. 2016;8(5):315. doi: 10.3390/nu8050315.

12. Zuraikat FM, Smethers AD, Rolls BJ. Potential moderators of the portion size effect. Physiol Behav. 2019;204:191-8. doi: 10.1016/j.physbeh.2019.02.043.

13. Mcclain A, van den Bos W, Matheson D, Desai M, Mcclure SM, Robinson TN. Visual illusions and plate design: The effects of plate rim widths and rim coloring on perceived food portion size. Int J Obes. 2014;38(5):657-62. doi: 10.1038/ ijo.2013.169.

14. González-Jiménez E, Río-Valle JS. Regulación de la ingesta alimentaria y del balance energético; factores y mecanismos implicados. Nutr Hosp. 2012;27(6):1850-9. doi: 10.3305/ nh.2012.27.6.6099.

15. Amin T, Mercer JG. Hunger and Satiety Mechanisms and Their Potential Exploitation in the Regulation of Food Intake. Curr Obes Rep. 2016;5(1):106-12. doi: 10.1007/s13679015-0184-5.

16. Ochoa C, Muñoz MG. Hambre, apetito y saciedad. Rev Cuba Aliment Nutr. 2014;24(2):268-79. 
17. García-Flores CL, Martínez MAG, Beltrán MCP, ZepedaSalvadorP, Solano Santos LV.Saciaciónvs saciedad: reguladores del consumo alimentario. Rev Med Chile. 2017;145(9):11728. doi: $10.4067 /$ s0034-98872017000901172.

18. Carranza QLE. Fisiología del apetito y el hambre. Enfermería Investiga, Investigación, Vinculación, Docencia y Gestión. 2016;1(3):117-24.

19. Palma JA, Iriarte J. Appetite regulation: Neuroendocrine basis and clinical approaches. Med Clin. 2012;139(2):70-5. doi: 10.1016/j.medcli.2011.11.024.

20. Yeung AY, Tadi P. Physiology, Obesity Neurohormonal Appetite and Satiety Control. En: StatPearls. Treasure Island; 2020.

21. Lim JJ, Poppitt SD. How satiating are the 'satiety' peptides: A problem of pharmacology versus physiology in the development of novel foods for regulation of food intake. Nutrients. 2019;11(7):1517. doi: 10.3390/nu11071517.

22. Behary P, Tharakan G, Alexiadou K, Johnson NA, Wewer ANJ, Cuenco J, et al. 57-OR: Combined GLP-1, Oxyntomodulin, and Peptide YY Improves Glycaemia and Body Weight in Obesity and Type 2 Diabetes: A Randomized, Single-Blinded Study. Diabetes Care. 2019;42(8):1446-1453. doi: 10.2337/ dc19-0449.

23. Izquierdo AG, Crujeiras $\mathrm{AB}$, Casanueva FF, Carreira MC. Leptin, obesity, and leptin resistance: where are we 25 years later? Nutrients. 2019;11(11):2704. doi: 10.3390/ nu11112704.

24. Gruzdeva O, Borodkina D, Uchasova E, Dyleva Y, Barbarash O. Leptin resistance: Underlying mechanisms and diagnosis. Diabetes Metab Syndr Obes. 2019;12:191-8. doi: 10.2147/ DMSO.S182406.

25. Borisenkov MF, Polugrudov AS, Paderin NM, Bakutova LA. Young inhabitants of the North with late chronotype and social jetlag consume more high-calorie foods and alcohol. Biol Rhythm Res. 2019;50(3):418-28. doi: 10.1080/09291016.2018.1455867.

26. St-Onge M-P, Pizinger T, Kovtun K, Roy Choudhury A. Sleep and meal timing influence food intake and its hormonal regulation in healthy adults with overweight/obesity. Eur J Clin Nutr. 2019;72(Suppl 1):76-82. doi: 10.1038/s41430-0180312-x.

27. King AJ, Burke LM, Halson SL, Hawley JA. The Challenge of Maintaining Metabolic Health During a Global Pandemic. Sport Med. 2020;50(7):1233-41. doi: 10.1007/s40279-02001295-8.

28. Nicolaidis S. Environment and obesity. Metabolism. 2019;100S:153942. doi: 10.1016/j.metabol.2019.07.006.

29. Tischmann L, Drummen M, Gatta-cherifi B, Raben A, Fogelholm M, Hartmann B, et al. Effects of a High-Protein / Moderate-Carbohydrate Diet on Appetite, Gut Peptides, and Endocannabinoids-A Preview Study. Nutrients. 2019;11(10):2269. doi: 10.3390/nu11102269.
30. McCrickerd K, Forde CG. Sensory influences on food intake control: Moving beyond palatability. Obes Rev. 2016;17(1):18-29. doi: 10.1111/obr.12340.

31. Yu S, Qualls-Creekmore E, Rezai-Zadeh K, Jiang Y, Berthoud $\mathrm{HR}$, Morrison CD, et al. Glutamatergic preoptic area neurons that express leptin receptors drive temperature-dependent body weight homeostasis. J Neurosci. 2016;36(18):5034-46. doi: 10.1523/JNEUROSCI.0213-16.2016.

32. Ruiz-Lozano T, Vidal J, de Hollanda A, Canteras M, Garaulet M, Izquierdo-Pulido M. Evening chronotype associates with obesity in severely obese subjects: Interaction with CLOCK 3111T/C. Int J Obes. 2016;40(10):1550-7. doi: 10.1038/ ijo.2016.116.

33. Mathiesen SL, Mielby LA, Byrne DV, Wang QJ. Music to eat by: A systematic investigation of the relative importance of tempo and articulation on eating time. Appetite. 2020;155:104801. doi: 10.1016/j.appet.2020.104801.

34. Ding L, Hamid N, Shepherd D, Kantono K. How is satiety affected when consuming food while working on a computer? Nutrients. 2019;11(7):1545. doi: https: 10.3390/ nu11071545.

35. Morquecho-Campos P, de Graaf K, Boesveldt S. Smelling our appetite? The influence of food odors on congruent appetite, food preferences and intake. Food Qual Prefer. 2020; 85:103959. doi: 10.1016/j.foodqual.2020.103959.

36. Sandberg JC, Björck IME, Nilsson AC. Effects of whole grain rye, with and without resistant starch type 2 supplementation, on glucose tolerance, gut hormones, inflammation and appetite regulation in an 11-14.5 hour perspective; a randomized controlled study in healthy subjects. Nutr J. 2017;16(1):25. doi: 10.1186/s12937-017-0246-5.

37. Poutanen KS, Dussort P, Erkner A, Fiszman S, Karnik K, Kristensen $\mathrm{M}$, et al. A review of the characteristics of dietary fibers relevant to appetite and energy intake outcomes in human intervention trials. Am J Clin Nutr. 2017;106(3):74754. doi: 10.3945/ajcn.117.157172.

38. Dagbasi A, Lett AM, Murphy K, Frost G. Understanding the interplay between food structure, intestinal bacterial fermentation and appetite control. Proc Nutr Soc. 2020:1-17. doi: $10.1017 /$ S0029665120006941.

39. Cruz-Requena M, Aguilar-González CN, Prado-Barragan LA, Carneiro-da Cunha M das G, dos Santos CMT, ContrerasEsquivel JC, et al. Dietary fiber: An ingredient against obesity. Emirates J Food Agric. 2016;28(8):522-30. doi: 10.9755/ ejfa.2015-07-521.

40. Stefoska-Needham A, Beck EJ, Tapsell LC. Perspective: The path to confirming and exploiting potential satietyenhancing effects of sorghum-based foods for human diets. Qual Assur Saf Crop Foods. 2016;8(4):593-6. doi: 10.3920/ QAS2015.0695.

41. Myers A, Gibbons C, Finlayson G, Blundell J. Associations among sedentary and active behaviours, body fat and appetite dysregulation: Investigating the myth of physical inactivity 
and obesity. Br J Sports Med. 2017;51(21):1540-44. doi: 10.1136/bjsports-2015-095640.

42. Dorling J, Broom DR, Burns SF, Clayton DJ, Deighton K, James LJ, et al. Acute and chronic effects of exercise on appetite, energy intake, and appetite-related hormones: The modulating effect of adiposity, sex, and habitual physical activity. Nutrients. 2018;10(9): 1140. doi: 10.3390/nu10091140.

43. Espel-Huynh HM, Muratore AF, Lowe MR. A narrative review of the construct of hedonic hunger and its measurement by the Power of Food Scale. Obes Sci Pract. 2018;4(3):238-49. doi: 10.1002/osp4.161.

44. Muscogiuri G, Barrea L, Annunziata G, Di Somma C, Laudisio D, Colao A, et al. Obesity and sleep disturbance: the chicken or the egg? Crit Rev Food Sci Nutr. 2019;59(13):2158-65. doi: $10.1080 / 10408398.2018 .1506979$.
45. Rao TP. Role of guar fiber in appetite control. Physiol Behav. 2016;164(Pt A): 277-83. doi: 10.1016/j.physbeh.2016.06.014.

46. Reid M, Maras JE, Shea S, Wood AC, Castro-Diehl C, Johnson DA, et al. Association between diet quality and sleep apnea in the Multi-Ethnic Study of Atherosclerosis. Sleep. 2019;42(1):zsy194. doi: 10.1093/sleep/zsy194.

47. Beaulieu K, Hopkins M, Blundell J, Finlayson G. Homeostatic and non-homeostatic appetite control along the spectrum of physical activity levels: An updated perspective. Physiol Behav. 2018;192:23-9. doi: 10.1016/j.physbeh.2017.12.032.

48. Gibbons C, Hopkins M, Beaulieu K, Oustric P, Blundell JE. Issues in Measuring and Interpreting Human Appetite (Satiety/Satiation) and Its Contribution to Obesity. Curr Obes Rep. 2019;8(2):77-87. doi: 10.1007/s13679-01900340-6. 